\title{
Off-branch polaritons and multiple scattering in semiconductor microcavities
}

\author{
P. G. Savvidis, ${ }^{1}$ C. Ciuti, ${ }^{2}$ J. J. Baumberg, ${ }^{1, *}$ D. M. Whittaker ${ }^{3}$ M. S. Skolnick,${ }^{4}$ and J. S. Roberts ${ }^{5}$ \\ ${ }^{1}$ Department of Physics \& Astronomy, University of Southampton, SO17 1BJ, United Kingdom \\ ${ }^{2}$ Physics Department, Swiss Federal Institute of Technology Lausanne, CH-1015 Lausanne-EPFL, Switzerland \\ ${ }^{3}$ Toshiba Research Europe Ltd, Cambridge, CB4 4WE, United Kingdom \\ ${ }^{4}$ Department of Physics, University of Sheffield, Sheffield S3 7RH, United Kingdom \\ ${ }^{5}$ Department of Electronic and Electrical Engineering, University of Sheffield, Sheffield, S1 3JD, United Kingdom
}

(Received 6 March 2001; revised manuscript received 3 May 2001; published 25 July 2001)

\begin{abstract}
Angle-resolved measurements show unexpected emission resonances of the polaritons in a semiconductor microcavity. These resonances appear when more than one in-plane polariton mode is macroscopically occupied. The new resonances observed in angle-resolved luminescence do not lie on the expected polariton branches and possess different dispersion relations with negative effective mass. The experimental results can be well explained using an interacting polariton model that treats multiple scattering. The $\mathbf{k}$ dispersion of the luminescence resonances is reproduced using the Bogolubov approximation that deals with the macroscopic coherence of the signal, pump, and idler modes. This model also explains many puzzling features such as the stimulation of resonant Rayleigh scattering in the backward direction. In addition, the use of nonresonant control beams for coherent control of the polaritons is shown both experimentally and theoretically. The rich complexity of new phenomena in optically excited semiconductor microcavities can be attributed to the distinctive anti-Hermitian or anomalous coupling between polaritons.
\end{abstract}

DOI: 10.1103/PhysRevB.64.075311

PACS number(s): 78.47.+p, 71.36.+c, 42.50.-p, 42.65. $-\mathrm{k}$

\section{INTRODUCTION}

Exciton polaritons are the normal modes of the strong light-matter coupling in semiconductor microcavities. ${ }^{1}$ Such half-light half-matter quasiparticles have sharply distorted energy dispersions and a pronounced nonlinear behavior, inherited from the cavity photon and quantum well exciton components, respectively. After the first nonlinear emission studies under nonresonant pumping, ${ }^{2,3}$ a significant acceleration of research has been devoted to the regime of resonant excitation. ${ }^{4-14}$ Indeed, the dynamics of interacting polariton particles can now be directly monitored using resonant optical pulses and performing angle-resolved measurements. Recently, huge polariton amplification has been seen in angleresolved ultrafast pump-probe experiments. ${ }^{4}$ Lower-branch polaritons with a specific in-plane wave vector $\mathbf{k}_{\mathbf{p}}$ are injected by a resonant pump pulse, while a probe weakly excites the $\mathbf{k}=0$ polariton mode. The probe beam has been found to stimulate the parametric conversion of two pump polaritons into the pair of polaritons at the wave vectors $\mathbf{0}$ (signal) and $\mathbf{2} \mathbf{k}_{\mathbf{p}}$ (idler). This process occurs for the "magic" wave vector $\mathbf{k}_{\mathrm{p}}$, which allows energy-momentum conservation for the polariton-polariton scattering. Under continuouswave excitation without a probe beam, the spontaneous activation of such a process has been observed, and very efficient parametric oscillation of the polariton modes has been achieved. ${ }^{8,12}$ In this situation, not only the pumped mode but also the signal and idler modes exhibit a macroscopic coherence, although the phase of each varies separately. The three polariton "condensates" can, in principle, drastically modify the spectral properties at other wave vectors and produce collective excitations with peculiar dispersions. A situation of this kind is seen in a very different system, namely, the Bose condensate in superfluid helium, whose collective excitations have been very successfully described by Bogolubov. ${ }^{15}$

In this paper, we present angle-resolved emission measurements from a semiconductor microcavity excited in this polariton parametric amplification regime. New polariton resonances are clearly resolved in spectral, temporal, and angular data. The extra emission branches are absent when the probe is switched off. Their energy is found to be off the normal polariton branches, and their dispersions are also anomalous. Within the model of interacting polaritons (treated as interacting bosons ${ }^{6,14}$ ), we successfully reproduce these experimental observations. The multiple wave mixing of the polaritons is found to reproduce the strong coherent emission at the wave vectors $\mathbf{k}=-\mathbf{k}_{\mathbf{p}}, \mathbf{3} \mathbf{k}_{\mathbf{p}}$ and explain the observed spectral structure. The angular dispersion of the incoherent luminescence can be explained by applying the Bogolubov approach to the polariton system in the presence of signal, pump, and idler "condensates." Five new pairpolariton branches arise in the present conditions, which can now intuitively explain most experiments. Perhaps the most interesting general feature of this paper is the contrast between the Hermitian coupling between photon and exciton, which gives rise to the polaritons, and the anomolous antiHermitian-like coupling between polariton pairs, which gives rise to the many anomalous features observed here. Anti-Hermitian or anomalous coupling, which occurs in parametrically interacting systems, is particularly easy to study here and, unlike the microwave version, ${ }^{16}$ naturally gives rise to a quasiparticle picture in optically driven microcavities.

In Sec. II we summarize the experimental conditions, and present the new data in Sec. III. The theory of multiple scattering is developed in Sec. IV and discussed in relation to the observations. Further experiments, varying the probe angle 
and showing coherent control, are detailed in Secs. V and VI, before we conclude.

\section{EXPERIMENTAL SETUP AND SAMPLE}

The semiconductor microcavity sample used in these experiments was grown by metalorganic vapor-phase epitaxy and is made of top (bottom) distributed Bragg reflectors (DBR's) consisting of 17 (20) alternating $\lambda / 4$ layers of GaAs $/ \mathrm{Al}_{0.18} \mathrm{Ga}_{0.82} \mathrm{As}$. Two pairs of three $100 \AA \mathrm{In}_{0.06} \mathrm{Ga}_{0.94} \mathrm{As}$ quantum wells are sandwiched between the DBR's and are placed at the two intracavity field antinodes. The optical cavity length is $\sim 3 \lambda_{e x} / 2$ and varies across the sample. The cavity mode energy is thus tunable by scanning the position on the sample used for measurements, allowing access to both positive and negative detunings, $\Delta=\omega_{e x}-\omega_{\text {cav }}$, of the cavity resonance at normal incidence, $\omega_{\text {cav }}(\theta=0)$, from the lowest exciton energy $\omega_{e x}$. For resonance at normal incidence $(\Delta=0)$ used in all these experiments, a normal-mode splitting of $\hbar \Omega \sim 7 \mathrm{meV}$ is achieved with lower polariton and upper polariton linewidths of $0.56 \mathrm{meV}$ and $1.2 \mathrm{meV}$, respectively.

The strong-coupling regime is achieved when the sample is placed inside a wide field-of-view cold-finger cryostat and is cooled to a temperature of $\sim 10 \mathrm{~K}$. Transform-limited ultrashort pulses, derived from a Ti:sapphire laser with a repetition rate of $76 \mathrm{MHz}$, are spectrally filtered by a spatial light modulator and grating arrangement to resonantly excite the lower polariton branch. Pump-probe measurements are performed in the transmission geometry and the light emitted by the sample in a general direction $\theta$, is collected in a cone of $\pm 0.14^{\circ}$ and is coupled into a multimode fiber. Polarization of the pump and probe beams is kept co-circular, to inject polaritons of one particular spin and avoid unwanted polarization effects that can affect the analysis. The pump incidence angle is $\theta_{p}=16.5^{\circ}$, while the probe is at normal incidence, $\theta=0^{\circ}$, unless otherwise stated. The relation between the incident angle $\theta$ and the in-plane wave vector $k$ is simply $k=(\omega / c) \sin \theta$, where $\omega$ is the optical frequency and $c$ the speed of light. Both pump and probe beams have angular widths of $\sim 1^{\circ}$. A liquid-nitrogen-cooled chargecoupled device and a monochromator with a spectral resolution of $0.035 \mathrm{meV}$, are used to analyze the time-integrated emitted light. Far-field images of the emission are acquired by projecting the emission on a screen placed behind the sample.

\section{EXPERIMENTAL RESULTS}

In the specific pump-probe geometry discussed above, the probe beam shows a net gain of 10 when the pump pulse arrives at the same time. When the probe is strong enough, the pump depletion is macroscopic and can be directly observed as a decrease of the incoherent luminescence around the pump angle. In this regime, we also observe sharp emission around the pump back-scattering angle, $\theta=-16.5^{\circ}$, seen as a new beam emerging from the sample as well as the idler (Fig. 1). Note that the relative emission intensity, recorded on these images at different angles, is only qualitative
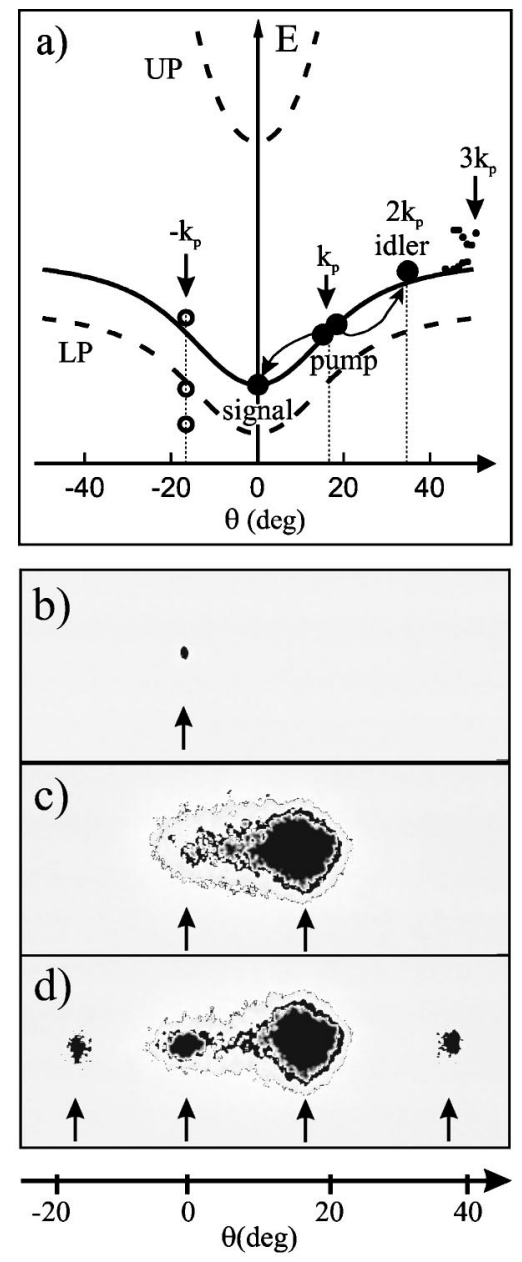

FIG. 1. (a) Unperturbed dispersion of the lower and upper polariton branch (dashed lines) and renormalized lower polariton branch (solid line). Arrows show the parametric conversion of pump polaritons $\left(\right.$ at $\left.16.5^{\circ}\right)$ into the probe $\left(0^{\circ}\right)$ and idler $\left(35^{\circ}\right)$ modes. The circles at $-16.5^{\circ}$ are the extracted peak positions of the emission spectra in Fig. 2(a). The points at large positive angles correspond to the peaks in Fig. 2(c). (b)-(d) Time integrated farfield images taken at pump-probe time delay $\tau=0$, for $2.4 \mathrm{~mW}$ pump power. (b) Probe only, (c) pump only, and (d) both pump and probe. New emission spots appear at the idler $35^{\circ}$ and the pump backscattering angle $-16.5^{\circ}$, as well as gain at signal $\theta=0^{\circ}$.

and depends on the camera angle at which the image was taken.

This emission spectrum at $-\mathbf{k}_{p}$ exhibits new energy peaks that exist clearly off the polariton branches and show dramatically nonlinear features. Figure 2(a) presents several emission spectra at $\theta=-16.5^{\circ}$, normalized to the pump power. At low pump intensities, the emission is peaked at the pump energy and has a linear dependence on the pump power. This is the well-known resonant Rayleigh scattering of the pump beam. When increasing the pump intensity, three new resonances appear in the spectrum with a strong superlinear dependence on the pump power. These features are completely absent when the probe beam is switched off (Fig. 2b). The energies of these peaks at $-\mathbf{k}_{p}$ are plotted in Fig. 1(a), together with the unperturbed dispersion of the 

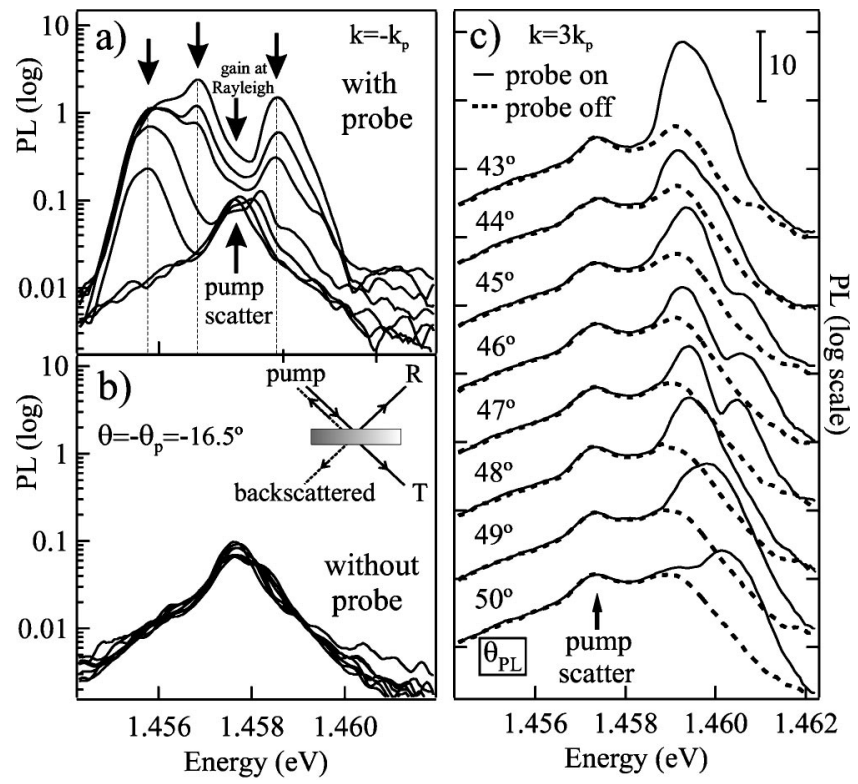

FIG. 2. (a) and (b) Emission as a function of pump power collected at $-16.5^{\circ}$, normalized to the pump power (increasing from $0.2 \mathrm{~mW}$ to $2.4 \mathrm{~mW}$ in steps of $0.4 \mathrm{~mW}$ ), both with and without probe. Inset shows the schematics of reflected $(R)$, transmitted $(T)$, and back-scattered (dashed) directions of the incident pump beam. (c) Normalized emission spectra, with and without probe in the angular range between $43^{\circ}$ and $50^{\circ}$, at $2.4 \mathrm{~mW}$ pump.

lower polariton branch. Remarkably, some of the measured peak energies are completely off branch.

The appearance of new emission resonances does not occur only at $\theta=-16.5^{\circ}$. Another relatively intense emission is shown in Fig. 2(c) for the angular range between $43^{\circ}$ and $50^{\circ}$. Switching on the probe produces additional peaks, which are clearly resolved [also plotted on Fig. 1(a)]. These enhancements at $-k_{p}, 0, k_{p}, 2 k_{p}$, and $3 k_{p}$ are maximized when the pump and probe arrive almost simultaneously (theoretically, the buildup time of the signal is seen to favor timing the probe to arrive on the leading edge of the pump pulse), and possess similar fast temporal responses controlled by the 5 ps lifetime of the photon component in the microcavity. Their complete dependence on the injected probe suggests that the macroscopic occupation of the signal and idler modes (whose occupation number $f$ becomes much larger than 1) is responsible for the observed emission. For a full picture, we have systematically measured the peak energies from the luminescence spectra in the whole range between $\pm 50^{\circ}$ (Fig. 3, with theoretical fits to be discussed later). The observed energies (points) clearly demonstrate the appearance of new emission branches whose energy dispersion is very peculiar. To proceed theoretically requires a full treatment of multiple polariton scattering.

\section{THEORETICAL ANALYSIS}

\section{A. Introduction}

The observed parametric amplification of the polariton modes has been successfully described within the interacting polariton model. The gain spectral, temporal, and angular

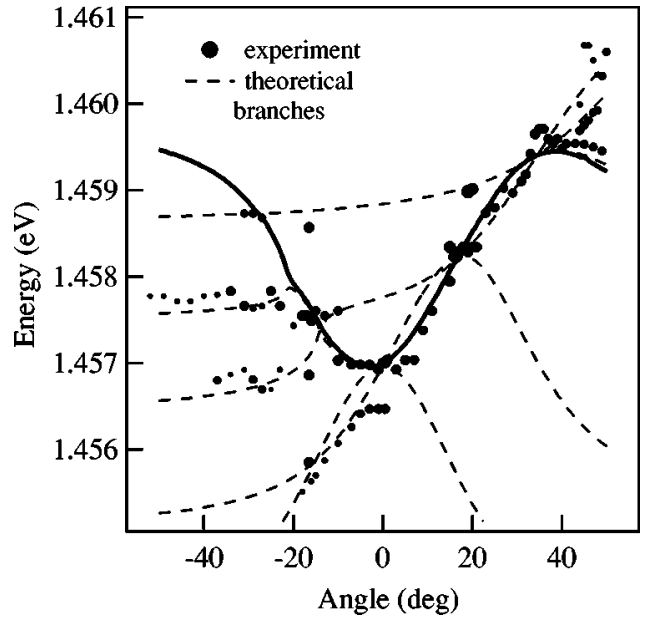

FIG. 3. Peak emission energy positions extracted from the time integrated spectra (circle size represents the logarithmic intensity of each peak). The dashed line shows the fitted multibranch energy dispersion.

properties have been very well reproduced through a set of three equations for the signal, pump, and idler modes. ${ }^{6,17}$ The dynamics predicted by the polariton Hamiltonian is indeed very reliable, encouraging an extension of the treatment to the multiple-scattering regime. In this section, we present the dynamical equations for the coherent occupation of the polariton modes. These equations involve all the in-plane wave vectors $\mathbf{k}$ and represent a generalization of the three mode equations for the polariton parametric amplifier.

Macroscopic occupation of three different $\mathbf{k}$ states along the dispersion introduces strong parametric coupling between polariton pairs which satisfy energy-momentum conservation. The restricted phasematching conditions result in new pair-polariton branches that are distinct from the original lower polariton dispersion. These new polariton branches are 'parametric mirrors' induced by the specific occupations of the microcavity. As we shall see, each branch is induced by a specific pair of polaritons.

\section{B. Coherent multiple scattering}

Before considering the dynamical equations, we briefly review the polariton Hamiltonian. ${ }^{6}$ When considering only the case of resonant pumping of the lower polariton branch, we can enormously simplify our description by neglecting the nonlinear contribution due to the upper branch, which is energetically well separated. The destruction operator for a lower polariton with in-plane wavevector $\mathbf{k}$ is $p_{\mathbf{k}}=X_{k} b_{\mathbf{k}}$ $+C_{k} a_{\mathbf{k}}$, where $b_{\mathbf{k}}$ and $a_{\mathbf{k}}$ are the exciton and photon Bose operators, respectively, and $X_{k}$ and $C_{k}$ the corresponding Hopfield coefficients $\left(X_{k}>0\right.$ and $\left.C_{k}<0\right)$. The lower polariton Hamiltonian is $H=H_{L P}+H_{P P}^{\text {eff }}+H_{q m}$. The free term $H_{L P}=\Sigma_{\mathbf{k}} E_{L P}(k) p_{\mathbf{k}}^{\dagger} p_{\mathbf{k}}$, contains the lower polariton energy dispersion $E_{L P}(k)$. The polariton-polariton interaction is due to the exciton-exciton interaction and exciton-photon saturation. Namely, we have 


$$
H_{P P}^{\mathrm{eff}}=\frac{1}{2} \sum_{\mathbf{k}, \mathbf{k}^{\prime}, \mathbf{q}} \frac{\lambda_{X}^{2}}{A} V_{\mathbf{k}, \mathbf{k}^{\prime}, \mathbf{q}}^{P P} p_{\mathbf{k}+\mathbf{q}}^{\dagger} p_{\mathbf{k}^{\prime}-\mathbf{q}}^{\dagger} p_{\mathbf{k}} p_{\mathbf{k}^{\prime}}
$$

where $\lambda_{X}$ is the two-dimensional exciton radius and $\mathrm{A}$ is the macroscopic quantization area. The interaction potential is $V_{\mathbf{k}, \mathbf{k}^{\prime}, \mathbf{q}}^{P P}=\left\{\left[2 \hbar \Omega_{R} / n_{s a t} \lambda_{X}^{2}\right]\left(\left|C_{\mathbf{k}+\mathbf{q}}\right| X_{\mathbf{k}^{\prime}}+\left|C_{\mathbf{k}^{\prime}}\right| X_{\mathbf{k}+\mathbf{q}}\right)\right.$ $\left.+\left(6 e^{2} / \epsilon \lambda_{X}\right) X_{\mathbf{k}+\mathbf{q}} X_{\mathbf{k}^{\prime}}\right\} X_{\mathbf{k}^{\prime}-\mathbf{q}} X_{\mathbf{k}}$, with $n_{\text {sat }}=7 /\left(16 \pi \lambda_{X}^{2}\right)$ the exciton saturation density, and $\epsilon$ the quantum well dielectric constant. Notice that $V_{\mathbf{k}, \mathbf{k}^{\prime}, \mathbf{q}}^{P P}$ is positive and represents a repulsive interaction. The cavity system interacts with the external electromagnetic field through the standard quasimode coupling Hamiltonian, $\quad H_{q m}=\int d \Omega\left\{\Sigma_{\mathbf{k}} g C_{\mathbf{k}} p_{\mathbf{k}}^{\dagger} \alpha_{\mathbf{k}, \Omega}\right.$ + H.c.\}. The operator $\alpha_{\mathbf{k}, \Omega}$ destructs an external photon with in-plane wavevector $\mathbf{k}$ and frequency $\Omega$. When an external optical field is applied, $\alpha_{\mathbf{k}}$ can be replaced by its mean value $\left\langle\alpha_{\mathbf{k}}\right\rangle$, which represents the classical value of the field.

An applied optical field with the in-plane wave vector $\mathbf{k}_{\mathbf{0}}$, drives a polarization $\left\langle p_{\mathbf{k}_{\mathbf{0}}}\right\rangle$ of the corresponding polariton mode. The coupled dynamics of every $\left\langle p_{\mathbf{k}}\right\rangle$ is given by the Heisenberg equations of motion generated by the interacting polariton Hamiltonian. Such equations become closed when the three-polariton expectation values are factorized in products of three-polariton polarizations. It is convenient to consider the rescaled quantity $\mathcal{P}_{\mathbf{k}}=\left(\lambda_{X} / \sqrt{A}\right)\left\langle p_{\mathbf{k}}\right\rangle$. The quantity $\left|\mathcal{P}_{\mathbf{k}}\right|^{2}$ represents the coherent density of polaritons in the $\mathbf{k}$ mode in units of $\lambda_{X}^{-2}$. The equation for $\mathcal{P}_{\mathbf{k}}$ reads

$$
\begin{aligned}
i \hbar \frac{d}{d t} \mathcal{P}_{\mathbf{k}}= & {\left[\widetilde{E}_{L P}(\mathbf{k})-i \gamma_{\mathbf{k}}\right] \mathcal{P}_{\mathbf{k}} } \\
& +\sum_{\mathbf{k}^{\prime}, \mathbf{k}^{\prime \prime} \neq \mathbf{k}} E_{\mathbf{k}, \mathbf{k}^{\prime}, \mathbf{k}^{\prime \prime}}^{i n t} \mathcal{P}_{\mathbf{k}^{\prime}+\mathbf{k}^{\prime \prime}-\mathbf{k}}^{\star} \mathcal{P}_{\mathbf{k}^{\prime}} \mathcal{P}_{\mathbf{k}^{\prime \prime}}+\mathcal{F}_{\mathbf{k}}(t)
\end{aligned}
$$

The coupling energy is defined as

$$
E_{\mathbf{k}, \mathbf{k}^{\prime}, \mathbf{k}^{\prime \prime}}^{i n t}=\frac{1}{2}\left(V_{\mathbf{k}^{\prime}, \mathbf{k}^{\prime \prime}, \mathbf{k}-\mathbf{k}^{\prime}}^{P P}+V_{\mathbf{k}^{\prime}, \mathbf{k}^{\prime \prime}, \mathbf{k}^{\prime \prime}-\mathbf{k}}^{P P}\right)
$$

The repulsive polariton interaction is responsible for the blueshift of the polariton branch seen in experiments, namely,

$$
\widetilde{E}_{L P}(\mathbf{k})=E_{L P}(k)+\sum_{\mathbf{k}^{\prime}} E_{\mathbf{k}, \mathbf{k}^{\prime}}^{\text {shift }}\left|\mathcal{P}_{\mathbf{k}^{\prime}}\right|^{2}
$$

where $E_{\mathbf{k}, \mathbf{k}^{\prime}}^{\text {shift }}=V_{\mathbf{k}, \mathbf{k}^{\prime}, \mathbf{0}}^{P P}+V_{\mathbf{k}, \mathbf{k}^{\prime}, \mathbf{k}^{\prime}-\mathbf{k}}^{P P}$. Finally, the applied external field produces the driving term $\mathcal{F}_{\mathbf{k}}(t) \propto g\left|C_{\mathbf{k}}\right|^{2} \Omega_{\mathbf{k}}(t)$, where $\Omega_{\mathbf{k}}(t)$ is the $\mathbf{k}$ component of the applied extracavity field.

Equation (2) actually describes the coherent multiple scattering of resonantly excited polaritons. The driving field $\mathcal{F}_{\mathbf{k}}(t)$ excites polaritons at specific wave vectors. In our configuration, the external field excites the wave vector $\mathbf{0}$ and $\mathbf{k}_{\mathbf{p}}$ (with a small angular width around each). Due to the polariton-polariton interaction, the coherent occupation at

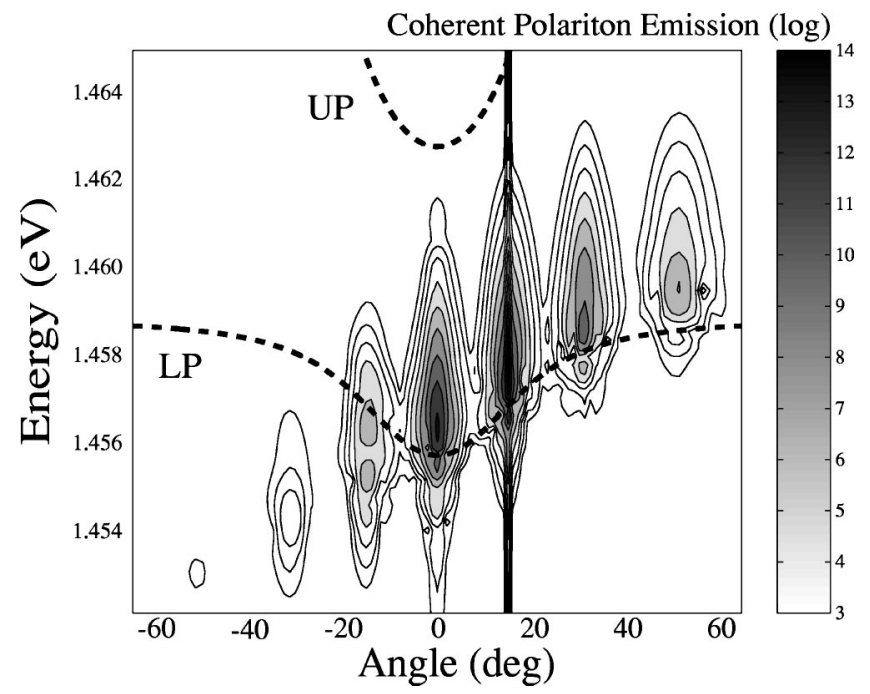

FIG. 4. Theoretical map of the coherent emission (log scale) as a function of the emission angle and energy. The pump and probe parameters correspond to the experimental configuration.

generic wave vectors $\mathbf{k}^{\prime}$ and $\mathbf{k}^{\prime \prime}$ can be scattered into the wave vectors $\mathbf{k}$ and $\mathbf{k}^{\prime}+\mathbf{k}^{\prime \prime}-\mathbf{k}$. As these wave vectors can scatter into other ones, this gives rise to a sort of multiplescattering cascade. As a first application, we have solved Eq. (2) by considering the excitation pulses and material parameters corresponding to the experiment. A contour plot of the quantity $\mathcal{T}_{\mathbf{k}}(\omega)=\left|C_{\mathbf{k}}\right|^{2}\left|\mathcal{P}_{\mathbf{k}}(\omega)\right|^{2}$ is plotted as a function of emission angle $\theta$ and energy $\hbar \omega$ (Fig. 4). $\mathcal{T}_{\mathbf{k}}(\omega)$ represents the polariton coherent density at the wave vector $\mathbf{k}$ and energy $\hbar \omega$, weighted by the photon fraction $\left|C_{\mathbf{k}}\right|^{2}$, and is proportional to the coherent transmission. Apart from the pump transmission, strong emission occurs at the signal and idler angles. Relatively intense emission beams are also observed around $-\mathbf{k}_{\mathbf{p}}$ and $\mathbf{3} \mathbf{k}_{\mathbf{p}}$. One component at $\mathbf{k}=-\mathbf{k}_{\mathbf{p}}$ is spectrally off branch and presents a well-developed splitting, which matches the experimental spectra at $\theta=-16.5^{\circ}$. Similarly the spot around $\mathbf{3} \mathbf{k}_{\mathbf{p}}$ accounts for the strong emission near $50^{\circ}$. The calculation also shows very weak emission around $\mathbf{k}=-\mathbf{2} \mathbf{k}_{\mathbf{p}}$, which is not resolved in the current experiment.

\section{Dispersion of the emission branches}

Equation (2) does not describe the luminescence but only the coherent wave mixing of the polariton matter. The problem of the polariton luminescence has been already treated in the case of a cw pump without probe in the regime below the threshold of the parametric oscillation. ${ }^{14}$ The luminescence is driven by the parametric correlation, which is stimulated by the incoherent polariton population. Generalizing to the case above threshold (with macroscopic occupations of signal and idler) represents a very complicated technical task, even though the physics is conceptually the same. However, the determination of the energy resonances of the polariton luminescence can be done in a quite a simple way following the Bogulobov approximation, which holds when a discrete set of polariton modes $S_{p o l}$ is coherently and 
macroscopically occupied. In our configuration, we can take $S_{p o l}=\left\{\mathbf{0}, \mathbf{k}_{\mathbf{p}}, \mathbf{2} \mathbf{k}_{\mathbf{p}}\right\}$. The Bogolubov approach essentially consists of two approximations. The first approximation is obtained by neglecting all scattering channels that do not have the macroscopically occupied modes as initial states. This means that we consider only the processes $\left\{\mathbf{k}^{\prime}, \mathbf{k}^{\prime \prime}\right\} \rightarrow\left\{\mathbf{k}, \mathbf{k}^{\prime}+\mathbf{k}^{\prime \prime}-\mathbf{k}\right\}$, where $\mathbf{k}^{\prime}, \mathbf{k}^{\prime \prime} \in S_{\text {pol }}$, that is they are macroscopically occupied. The second step consists in neglecting the quantum fluctuations of these macroscopically occupied modes. Mathematically, this corresponds to the approximation $p_{\mathbf{k}}^{\dagger}(t) p_{\mathbf{k}^{\prime}+\mathbf{k}^{\prime \prime}-\mathbf{k}}^{\dagger}(t) p_{\mathbf{k}^{\prime}}(t) p_{\mathbf{k}^{\prime \prime}}(t)$ $\simeq p_{\mathbf{k}}^{\dagger}(t) p_{\mathbf{k}^{\prime}+\mathbf{k}^{\prime \prime}-\mathbf{k}}^{\dagger}(t)\left\langle p_{\mathbf{k}^{\prime}}(t)\right\rangle\left\langle p_{\mathbf{k}^{\prime \prime}}(t)\right\rangle$. With these assumptions, the equation of motion for the polariton operator $p_{\mathbf{k}}(t)$ reads

$$
\begin{aligned}
i \hbar \frac{d}{d t} p_{\mathbf{k}}(t)= & {\left[\widetilde{E}_{L P}(\mathbf{k})-i \gamma_{k}\right] p_{\mathbf{k}}(t)+F_{\mathbf{k}}(t) } \\
& +\sum_{\mathbf{k}^{\prime}, \mathbf{k}^{\prime \prime} \in S_{p o l}} E_{\mathbf{k}, \mathbf{k}^{\prime}, \mathbf{k}^{\prime \prime}}^{i n t} \mathcal{P}_{\mathbf{k}^{\prime}}(t) \mathcal{P}_{\mathbf{k}^{\prime \prime}}(t) p_{\mathbf{k}^{\prime}+\mathbf{k}^{\prime \prime}-\mathbf{k}}^{\dagger}(t) .
\end{aligned}
$$

The coupling to the external electromagnetic field is responsible, not only for the radiative loss $\gamma_{k}$, but also for a quantum fluctuation Langevin force $F_{\mathbf{k}}(t)$ $=\int d \Omega g C_{\mathbf{k}}^{\star} e^{-i \Omega t} \alpha_{\mathbf{k}, \Omega}(0)$. Finally, the evolution of the macroscopically occupied modes can be approximated with the expression $\overline{\mathcal{P}}_{\mathbf{k}^{\prime}} \exp \left[-(i / \hbar) \widetilde{E}_{L P}\left(\mathbf{k}^{\prime}\right) t\right]$, where $\overline{\mathcal{P}}_{\mathbf{k}^{\prime}}$ is a slowly varying function of time.

The polariton operator $p_{\mathbf{k}}(t)$ is coupled to the operator $p_{\mathbf{k}^{\prime}+\mathbf{k}^{\prime \prime}-\mathbf{k}}^{\dagger}(t)$, where $\mathbf{k}^{\prime}, \mathbf{k}^{\prime \prime} \in S_{\text {pol }}$. The wave vector $\mathbf{k}^{\prime}+\mathbf{k}^{\prime \prime}$ $-\mathbf{k}$ corresponds to the process

$$
\left\{\mathbf{k}^{\prime}, \mathbf{k}^{\prime \prime}\right\} \rightarrow\left\{\mathbf{k}, \mathbf{k}^{\prime}+\mathbf{k}^{\prime \prime}-\mathbf{k}\right\}
$$

For the particular set $S_{p o l}=\left\{\mathbf{0}, \mathbf{k}_{\mathbf{p}}, \mathbf{2} \mathbf{k}_{\mathbf{p}}\right\}, p_{\mathbf{k}}(t)$ is coupled to 5 wave vectors $\mathbf{k}_{\mathbf{p p}}, \mathbf{k}_{\mathrm{sp}}, \mathbf{k}_{\mathbf{p i}}, \mathbf{k}_{\mathbf{s s}}$, and $\mathbf{k}_{i i}$, each of which correspond to different processes (Fig. 5). Namely,

(i) pump-pump scattering is $\left\{\mathbf{k}_{\mathbf{p}}, \mathbf{k}_{\mathbf{p}}\right\} \rightarrow\left\{\mathbf{k}, \mathbf{k}_{\mathbf{p} \mathbf{p}}\right\}$, with $\mathbf{k}_{\mathbf{p p}}$ $=2 \mathbf{k}_{\mathbf{p}}-\mathbf{k}$. The condition of energy conservation is $\widetilde{E}_{L P}(\mathbf{k})$ $=E_{p p}(\mathbf{k})$, where the pump-pump branch is defined as

$$
E_{p p}(\mathbf{k})=2 \widetilde{E}_{L P}\left(\mathbf{k}_{\mathbf{p}}\right)-\widetilde{E}_{L P}\left(\mathbf{k}_{\mathbf{p p}}\right) .
$$

(ii) signal-pump scattering is $\left\{\mathbf{0}, \mathbf{k}_{\mathbf{p}}\right\} \rightarrow\left\{\mathbf{k}, \mathbf{k}_{\mathbf{s p}}\right\}$, with $\mathbf{k}_{\mathbf{s p}}$ $=\mathbf{k}_{\mathbf{p}}-\mathbf{k}$. The corresponding branch reads

$$
E_{s p}(\mathbf{k})=\widetilde{E}_{L P}(\mathbf{0})+\widetilde{E}_{L P}\left(\mathbf{k}_{\mathbf{p}}\right)-\widetilde{E}_{L P}\left(\mathbf{k}_{\mathbf{s p}}\right) .
$$

(iii) pump-idler scattering is $\left\{\mathbf{k}_{\mathbf{p}}, 2 \mathbf{k}_{\mathbf{p}}\right\} \rightarrow\left\{\mathbf{k}, \mathbf{k}_{\mathbf{p} \mathbf{i}}\right\}$, with $\mathbf{k}_{\mathbf{p i}}$ $=3 \mathbf{k}_{\mathbf{p}}-\mathbf{k}$. The related branch

$$
E_{p i}(\mathbf{k})=\widetilde{E}_{L P}\left(\mathbf{2} \mathbf{k}_{\mathbf{p}}\right)+\widetilde{E}_{L P}\left(\mathbf{k}_{\mathbf{p}}\right)-\widetilde{E}_{L P}\left(\mathbf{k}_{\mathbf{p i}}\right) .
$$

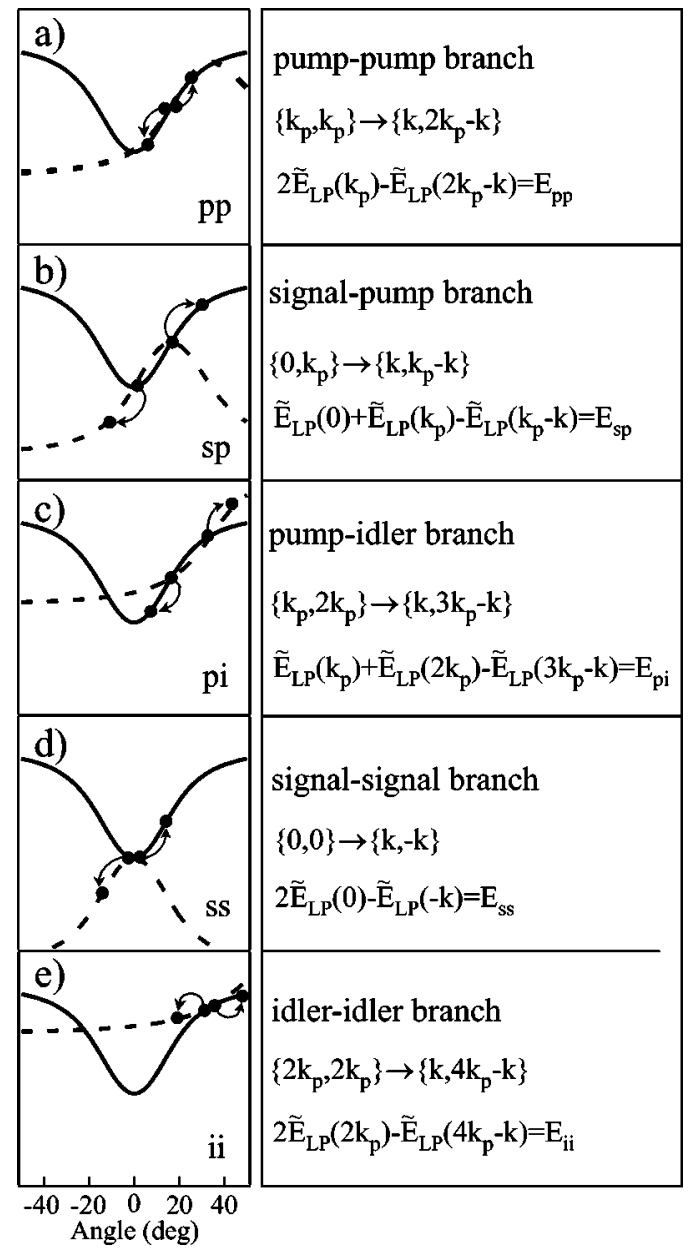

FIG. 5. Scattering channels starting from two polaritons in macroscopically occupied states and their corresponding induced wavemixing branches (dashed).

(iv) signal-signal scattering is $\{\mathbf{0}, \mathbf{0}\} \rightarrow\left\{\mathbf{k}, \mathbf{k}_{\mathrm{ss}}\right\}$, with $\mathbf{k}_{\mathrm{ss}}=$ $-\mathbf{k}$. The signal-signal branch dispersion reads

$$
E_{s s}(\mathbf{k})=2 \widetilde{E}_{L P}(\mathbf{0})-\widetilde{E}_{L P}\left(\mathbf{k}_{\mathrm{ss}}\right) .
$$

(v) idler-idler scattering is $\left\{2 \mathbf{k}_{\mathbf{p}}, 2 \mathbf{k}_{\mathbf{p}}\right\} \rightarrow\left\{\mathbf{k}, \mathbf{k}_{\mathbf{i i}}\right\}$, with $\mathbf{k}_{\mathbf{i i}}$ $=4 \mathbf{k}_{\mathbf{p}}-\mathbf{k}$. The corresponding energy branch has the dispersion

$$
E_{i i}(\mathbf{k})=2 \widetilde{E}_{L P}\left(\mathbf{2} \mathbf{k}_{\mathbf{p}}\right)-\widetilde{E}_{L P}\left(\mathbf{k}_{\mathrm{ii}}\right) .
$$

The full set of scattering processes and the corresponding wave-mixing dispersion branches are depicted in Fig. 5. Finally, we point out that the signal-idler scattering gives rise to the same branch as the pump-pump scattering, because $\widetilde{E}_{L P}(\mathbf{0})+\widetilde{E}_{L P}\left(\mathbf{2} \mathbf{k}_{\mathbf{p}}\right)=2 \widetilde{E}_{L P}\left(\mathbf{k}_{\mathbf{p}}\right)$.

In order to go further, it is convenient to define the following vector of polariton operators 


$$
v_{\mathbf{k}}(t)=\left(\begin{array}{c}
p_{\mathbf{k}}(t) \\
p_{\mathbf{k}_{\mathbf{p p}}}^{\dagger}(t) \exp \left\{-\frac{i}{\hbar}\left[E_{p p}(\mathbf{k})+\widetilde{E}_{L P}\left(\mathbf{k}_{\mathbf{p p}}\right)\right] t\right\} \\
p_{\mathbf{k}_{\mathbf{s p}}^{\dagger}}^{\dagger}(t) \exp \left\{-\frac{i}{\hbar}\left[E_{s p}(\mathbf{k})+\widetilde{E}_{L P}\left(\mathbf{k}_{\mathbf{s p}}\right)\right] t\right\} \\
p_{\mathbf{k}_{\mathbf{p i}}}^{\dagger}(t) \exp \left\{-\frac{i}{\hbar}\left[E_{p i}(\mathbf{k})+\widetilde{E}_{L P}\left(\mathbf{k}_{\mathbf{p i}}\right)\right] t\right\} \\
p_{\mathbf{k}_{\mathbf{s s}}}^{\dagger}(t) \exp \left\{-\frac{i}{\hbar}\left[E_{s s}(\mathbf{k})+\widetilde{E}_{L P}\left(\mathbf{k}_{\mathbf{s s}}\right)\right] t\right\} \\
p_{\mathbf{k}_{\mathbf{i i}}}^{\dagger}(t) \exp \left\{-\frac{i}{\hbar}\left[E_{i i}(\mathbf{k})+\widetilde{E}_{L P}\left(\mathbf{k}_{\mathbf{i i}}\right)\right] t\right\}
\end{array} \mid,\right.
$$

and a vector of Langevin forces

$$
\hat{F}_{\mathbf{k}}(t)=\left\{\begin{array}{l}
F_{\mathbf{k}}(t) \\
-F_{\mathbf{k}_{\mathbf{p p}}}^{\dagger}(t) \exp \left\{-\frac{i}{\hbar}\left[E_{p p}(\mathbf{k})+\widetilde{E}_{L P}\left(\mathbf{k}_{\mathbf{p p}}\right)\right] t\right\} \\
-F_{\mathbf{k}_{\mathbf{s p}}}^{\dagger}(t) \exp \left\{-\frac{i}{\hbar}\left[E_{s p}(\mathbf{k})+\widetilde{E}_{L P}\left(\mathbf{k}_{\mathbf{s p}}\right)\right] t\right\} \\
-F_{\mathbf{k}_{\mathbf{p i}}}^{\dagger}(t) \exp \left\{-\frac{i}{\hbar}\left[E_{p i}(\mathbf{k})+\widetilde{E}_{L P}\left(\mathbf{k}_{\mathbf{p i}}\right)\right] t\right\} \\
-F_{\mathbf{k}_{\mathbf{s s}}}^{\dagger}(t) \exp \left\{-\frac{i}{\hbar}\left[E_{s s}(\mathbf{k})+\widetilde{E}_{L P}\left(\mathbf{k}_{\mathbf{s s}}\right)\right] t\right\} \\
-F_{\mathbf{k}_{\mathbf{i i}}}^{\dagger}(t) \exp \left\{-\frac{i}{\hbar}\left[E_{i i}(\mathbf{k})+\widetilde{E}_{L P}\left(\mathbf{k}_{\mathbf{i i}}\right)\right] t\right\}
\end{array} \mid .\right.
$$

This way, the polariton dynamics are described by the operator system of equations

$$
i \hbar \frac{d}{d t} v_{\mathbf{k}}(t)=\mathcal{M}_{\mathbf{k}} v_{\mathbf{k}}(t)+\hat{F}_{\mathbf{k}}(t),
$$

where the sparse matrix $\mathcal{M}_{\mathbf{k}}$ is a non-Hermitian matrix. The diagonal elements of $\mathcal{M}_{\mathbf{k}}$ are the wave-mixing branch energy dispersions, namely,

$$
\begin{aligned}
& \left(\mathcal{M}_{\mathbf{k}}\right)_{11}=\widetilde{E}_{L P}(\mathbf{k}), \\
& \left(\mathcal{M}_{\mathbf{k}}\right)_{22}=E_{p p}(\mathbf{k}), \\
& \left(\mathcal{M}_{\mathbf{k}}\right)_{33}=E_{s p}(\mathbf{k}), \\
& \left(\mathcal{M}_{\mathbf{k}}\right)_{44}=E_{p i}(\mathbf{k}), \\
& \left(\mathcal{M}_{\mathbf{k}}\right)_{55}=E_{s s}(\mathbf{k}), \\
& \left(\mathcal{M}_{\mathbf{k}}\right)_{66}=E_{i i}(\mathbf{k}) .
\end{aligned}
$$

The ordinary polariton branch $\widetilde{E}_{L P}(\mathbf{k})$ is directly coupled to the other five ones. The corresponding off-diagonal matrix elements are

$$
\begin{gathered}
\left(\mathcal{M}_{\mathbf{k}}\right)_{12}=E_{\mathbf{k}, \mathbf{k}_{\mathbf{p}}, \mathbf{k}_{\mathbf{p}}}^{i n t} \overline{\mathcal{P}}_{\mathbf{k}_{\mathbf{p}}}^{2}+2 E_{\mathbf{k}, \mathbf{0}, \mathbf{2} \mathbf{k}_{\mathbf{p}}}^{i n t} \overline{\mathcal{P}}_{\mathbf{0}} \overline{\mathcal{P}}_{2 \mathbf{k}_{\mathbf{p}}} \\
\left(\mathcal{M}_{\mathbf{k}}\right)_{13}=2 E_{\mathbf{k}, \mathbf{0}, \mathbf{k}_{p}}^{i n t} \overline{\mathcal{P}}_{\mathbf{0}} \overline{\mathcal{P}}_{\mathbf{k}_{\mathbf{p}}}, \\
\left(\mathcal{M}_{\mathbf{k}}\right)_{14}=2 E_{\mathbf{k}, \mathbf{k} \mathbf{p}, 2 \mathbf{k}_{\mathbf{p}}}^{i n t} \overline{\mathcal{P}}_{\mathbf{k}_{\mathbf{p}}} \overline{\mathcal{P}}_{2 \mathbf{k}_{\mathbf{p}}}, \\
\left(\mathcal{M}_{\mathbf{k}}\right)_{15}=E_{\mathbf{k}, \mathbf{0}, \mathbf{0}}^{i n t} \overline{\mathcal{P}}_{\mathbf{0}}^{2}, \\
\left(\mathcal{M}_{\mathbf{k}}\right)_{16}=2 E_{\mathbf{k}, \mathbf{2} \mathbf{k}_{\mathbf{p}}, 2 \mathbf{k}_{\mathbf{p}}}^{i n t} \overline{\mathcal{P}}_{2 \mathbf{k}_{\mathbf{p}}}^{2}
\end{gathered}
$$

\section{Anti-Hermitian or anomalous coupling}

The off-diagonal elements of the coupling matrix satisfy the relation

$$
\left(\mathcal{M}_{\mathbf{k}}\right)_{1 n}=-\left(\mathcal{M}_{\mathbf{k}}\right)_{n 1}^{\star},
$$

which is characteristic of the parametric or anti-Hermitian or anomalous coupling. All the other matrix elements of $\left(\mathcal{M}_{\mathbf{k}}\right)$ are zero. Such a system of operatorial equations is the starting point to calculate many-operator expectation values that provide access to experimental observables such as the photoluminescence. To obtain the energy resonances of the emission spectra, we simply have to find the eigenvalues of the complex matrix $\mathcal{M}_{\mathbf{k}}$, which are shown as dashed lines in Fig. 3, overlaying and in excellent agreement with the experimental data. Since this matrix has been obtained by performing a cw approximation, the comparison with the pulsed experiment has to be done by fitting an average value for the pump, signal, and idler coherent densities $\left|\mathcal{P}_{\mathbf{k}_{\mathbf{p}}}\right|^{2},\left|\mathcal{P}_{\mathbf{0}}\right|^{2}$, and $\left|\mathcal{P}_{\mathbf{2 k}_{\mathbf{p}}}\right|^{2}$. The total density is constrained by the observed blueshift of the original lower polariton branch. A good fit is obtained by using a signal to pump density ratio $\left|\mathcal{P}_{\mathbf{0}}\right|^{2} /\left|\mathcal{P}_{\mathbf{2} \mathbf{k}_{\mathrm{p}}}\right|^{2} \simeq 0.08$ and an idler to pump ratio $\left|\mathcal{P}_{\mathbf{0}}\right|^{2} /\left|\mathcal{P}_{\mathbf{2 k}_{\mathbf{p}}}\right|^{2}$ $\simeq 0.04$. These values are consistent with the time-resolved solutions of the coherent wave mixing whose spectra are shown in Fig. 4.

Because of the anomalous coupling [Eq. (9)], the behavior of the eigenvalues is very different to the case of Hermitian coupling. The situation is schematically depicted in Fig. 6. In the case of a matrix with Hermitian coupling, two quasiresonant levels anticross. With increasing interaction, the real part of the eigenvalues repel and at the same time, the imaginary (damping) parts tend to a mean value. This is the classical anticrossing between two coupled damped harmonic oscillators, and is exactly the situation for the coupling of the excitons and photons in the microcavity. In the case of antiHermitian coupling for the off-diagonal terms, the behavior is opposite. With increasing interaction, the real parts of the eigenvalues attract, while the imaginary parts split apart. This means that one mode is overdamped, while the other one is narrowed and can undergo amplification. The antiHermitian coupling is characteristic of parametric or 'anomalous' coupling between two oscillators.

The branches in Fig. 3 qualitatively reproduce the dispersive character of the resonances extracted from the luminescence. The emission resonances have a very asymmetric dis- 


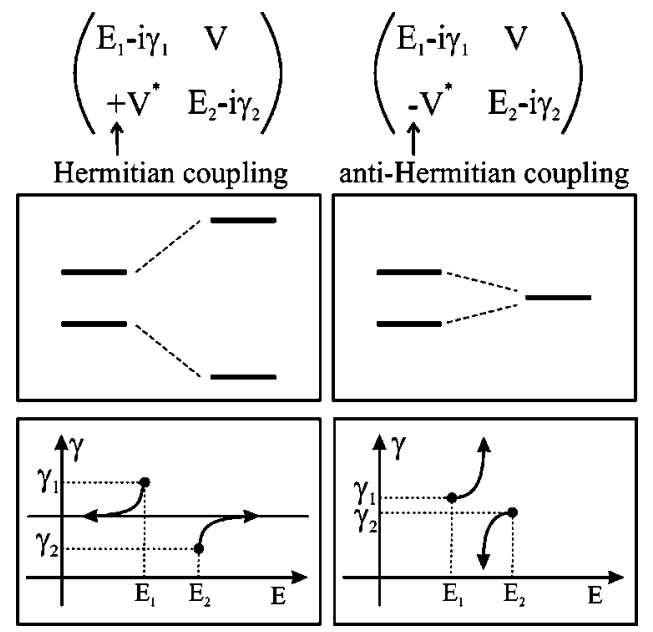

FIG. 6. Comparison of Hermitian and anomolous coupling. For Hermitian coupling, levels repel, and their imaginary (damping) parts become equal. For anti-Hermitian-like coupling, levels attract and their imaginary parts repel with one of the levels becoming overdamped and the other exhibiting gain when the imaginary part is negative.

persion and are completely off the branches of the unperturbed lower polariton dispersion. They are not exactly at the resonant Rayleigh scattering energy either, as suggested in recent experiments. ${ }^{7,18}$ Polariton branches, corresponding to different scattering channels cross and antiHermitian coupling, leads to their attraction and the flattening of the dispersion in that region (as for the measured dispersion around $\mathbf{k}=\mathbf{0}$, Fig. 3). The emission is expected to be particularly intense at these crossing points between branches. The branch, corresponding to the pumppump scattering channel, completely overlaps with the perturbed polariton dispersion in the entire region between $0^{\circ}$ and $35^{\circ}$ [Fig. 5(a)] and is responsible for the strong parametric luminescence observed in the absence of a probe beam. ${ }^{10}$ On the other hand, its crossing with the branch corresponding to the pump-signal scattering channel, is responsible for the bright emission spot at the pump back-scattering angle of $-16.5^{\circ}$ degrees, seen in Fig. 2. This is the origin of the enhanced coherent backscatter observed universally in semiconductor microcavities. In addition, the crossing point between the pump-signal and signal-signal branches is also exactly at the pump back-scattering angle of $-16.5^{\circ}$, and results in the buildup of efficient low-energy off-branch emission at that angle.

The lower-energy emission peak at $\mathbf{k}=\mathbf{0}$, seen in the experiment (Fig. 3), and seen as a shoulder in the full theory (Fig. 4), is produced by subsequent scattering from the - kp mode. In the multiple-wave-mixing equations, the multiple-scattering is accounted for at all orders. However in the matrix approach, we have truncated the multiplescattering cascade by considering only the channels having pump, signal, and idler as initial states. By including macroscopic populations also at $-\mathbf{k p}$ and $3 \mathbf{k p}$, we would find a next generation of additional channels. However at wave vectors $\mathbf{k}<0$, many of the new pair-polariton modes from the matrix approach are clearly visible in the experiments and account for the much stronger emission seen at negative angles. ${ }^{10}$ Despite the evident success of this dispersion mode analysis, we are unable, at the present stage, to calculate the precise intensity of luminescence from the different branches. Such issues will be addressed elsewhere. It is clear, however, that where modes cross, the emission is substantially enhanced.

We also find a vanishing or even negative branch dispersion around $\theta=35^{\circ}$. This suggests that it is possible to immobilize (or bring to a dead stop) polaritons in that region, or even in the case of negative dispersion, to reverse the direction of their propagation. This corresponds to a light-induced negative effective mass for the polaritons! The ability to use injected pulses to control the mass of polaritons, indicates the possibility of controlling their wave packets subsequent to their excitation. The analysis of the wave-packet propagation of signal, idler, and off-branch polaritons will also be left to another publication.

\section{ANGULAR DISPERSION OF STIMULATED GAIN}

From the preceding analysis, it is clear that coherent macroscopic occupations at different points on the dispersion relation, modify the quasiparticle dispersion. More specifically, the polariton-polariton interaction introduces local distortions and nonlocal changes to the dispersion with the appearance of new branches that are theoretically predicted and experimentally observable.

In this section we demonstrate that such multiple scattering is not limited to the particular pump-probe configuration discussed so far. The distortions of the occupied dispersion relation provide an explanation of why the probe gain can be observed even when energy-momentum conservation should not allow scattering to signal and idler. In addition, the data show that elastic scattering of pump photons (that breaks $k$ conservation) can be stimulated. These results can be attributed to the off-branch scattering developed above.

The experimental conditions are modified by allowing the probe angle to vary while keeping the pump angle fixed at $\theta_{p}$. Instead of the expected narrow range of probe angles, which can evoke a stimulated response, the injection of a small polariton population is found to affect the polariton scattering at most angles. Figure 7 shows emission spectra taken in the probe transmission direction, when both pump and probe beams are incident at the same $\tau=0$ time delay. In addition, pump photoluminesce (PL) and linear probe transmission spectra are recorded when each beam is individually blocked. The linear transmission spectra (dashed) agree well with the transfer-matrix model, yielding dispersivelike features at large incident angles. The pump luminescence spectra (thin lines) in the regime of parametric polariton scattering, are well described by the recently developed theory, ${ }^{14}$ in which injection of a large polariton population rigidly blueshifts the entire lower polariton dispersion. The extracted peak emission energies for the PL and the probe gain are plotted in Fig. $8(\mathrm{a}, \times)$ and show good agreement with this rigid shift (which is of the order of the polariton linewidth).

However the probe is found to be amplified for a large range of probe angles [Fig. 8(b)] at different energies. That stimulated scattering does not just occur to the ground state 


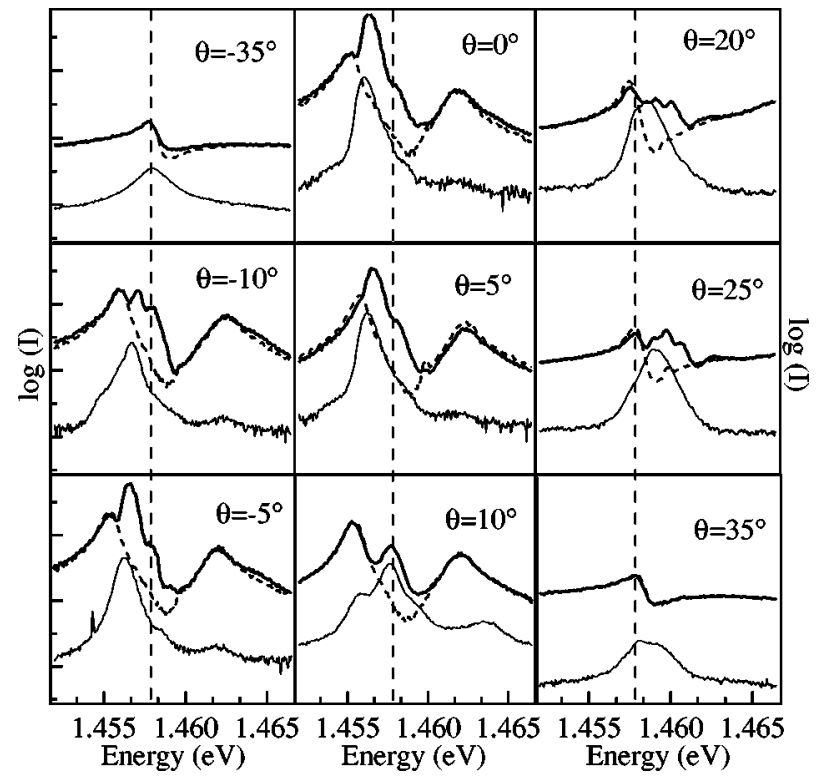

FIG. 7. Spectra taken in transmission geometry for the range of probe angles shown, pump $\theta_{p}=16.5^{\circ}$ held constant. PL spectra of the pump alone (thin), linear probe transmission spectra without pump (dashed), and multistructured gain peaks observed when both pump and probe beam are incident at the same time delay (solid). The pump and probe powers are $1 \mathrm{~mW}$ and $70 \mu \mathrm{W}$. Each vertical tick corresponds to a tenfold increase in intensity.

of the lower polariton branch, but is due to the distortion of the dispersion relation from multiple scattering, which produces extra solutions for energy and momentum conservation. This is also responsible for a number of new gain peaks that are clearly observed at higher energies [Fig. 8(a)]. Perhaps most surprising is the observation that the elastic scattering at the pump energy (dashed vertical lines in Fig. 7) is strongly enhanced by the injection of a weak probe pulse. The extracted positions of this stimulated peak ( $\boldsymbol{\Delta}$ in Fig. 8) confirm that this signal is degenerate with the pump wavelength. Rayleigh scattering, which breaks in-plane momentum conservation but conserves photon energy, is normally attributed to scattering off disorder within the quantum well in-plane potential. To be invoked for the results observed, such a model would suggest the unlikely scenario that inplane disorder is amplified by the occupation of the largediameter polariton states. Instead we suggest this effect arises from pump-signal scattering [Fig. 5(b)] in which neardegenerate scattering of the signal on the flattened bottom of the polariton dispersion (Fig. 3) causes near-degenerate scattering of the pump. Further theoretical and experimental work is in progress to test this suggestion. Once again, however, time-resolved spectroscopy allows direct observation of multiple scattering, which is smeared out and difficult to disentangle in cw experiments.

\section{COHERENT CONTROL THROUGH OFF-BRANCH EXCITATION}

The effect of macroscopically coherent populations, positioned along the dispersion relation, has a fundamental effect
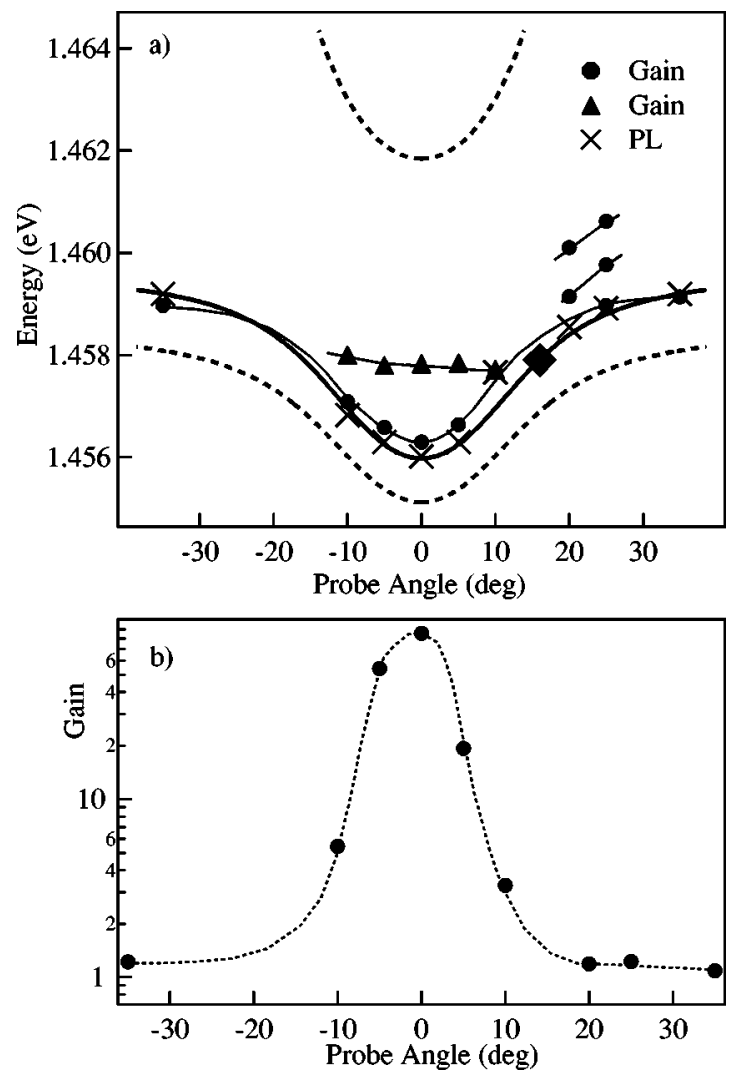

FIG. 8. (a) Upper and lower polariton branches (dashed) measured from the probe linear transmission spectra. The pump PL peak emission positions $(X)$ follow the parametric luminescence model, however the gain in the probe transmission direction $(0)$, extracted from Fig. 7, does not. Gain is also seen at the resonant Rayleigh scattering energy ( $\boldsymbol{\Delta}$ ). (b) Extracted maximum probe gain vs probe incident angle for constant pump angle $16.5^{\circ}$.

in producing new pair-polariton modes. These off-branch features are not only significant in emission, but also in excitation. We demonstrate this by showing that the spectrum of the pump pulse is crucial in controlling the multiple scattering.

Normally, due to the filtering effect of the microcavity, little difference is expected when pumping with a $100 \mathrm{fs}$ pulse $(10 \mathrm{~nm}$ bandwidth, which is tuned to overlap only with the lower polariton), and a $5 \mathrm{ps}$ pulse $(0.2 \mathrm{~nm}$ bandwidth, matched to the lower polariton linewidth). This assumes that light, which is not resonant with the lower polariton, merely reflects off the microcavity. ${ }^{19}$ However, due to the multiple scattering that produces new off-branch modes, we find that this is not the case, and the two different excitation conditions produce very different results. To simplify our experiment, we spectrally divide the pump pulse into a 5 ps pulse, which is resonant with the lower polariton branch, and a second higher-energy 5 ps ("control") pulse. We limit ourselves to the case where the control pulse is tuned to 1.4618 $\mathrm{eV}$, halfway in-between the upper and lower polaritons at this angle, which minimizes possible overlap with the bare polariton branches. Surprisingly, the off-resonant control pulse decreases the parametric gain of the signal (Fig. 9). More surprisingly, the idler is nearly completely suppressed-the 


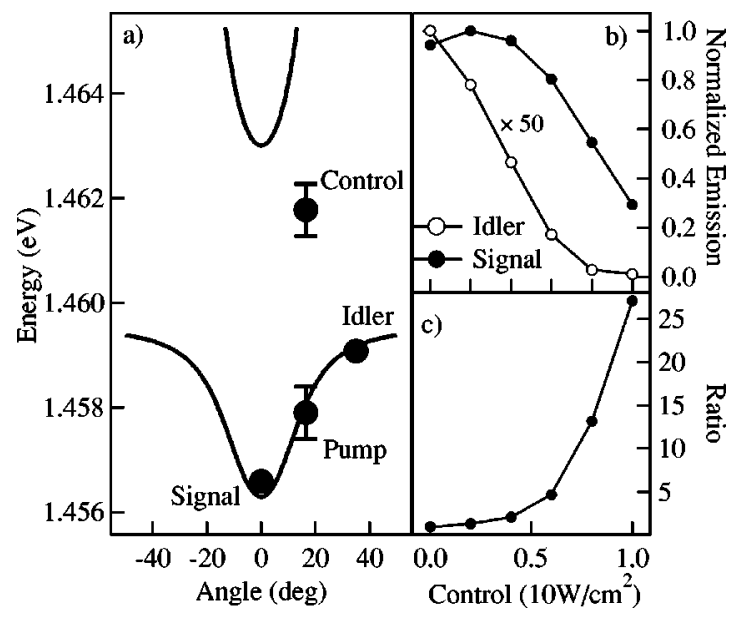

FIG. 9. (a) Coherent control: a second control pump beam is simultaneously applied at a higher nonresonant energy, opening an efficient scattering channel for the idler polaritons. The pump and control powers are $2 \mathrm{~mW}$ and $1 \mathrm{~mW}$. (b) Strength of signal and idler emission and (c) their ratios, as a function of the control pulse power.

relative suppression ratio for the signal and the idler beams can be as high as 25 . This method of coherently controlling the emission in different directions by applying a control beam, can provide an effective means of manipulating the polariton populations and can possibly be used for switching applications.

To study this kind of process, we have calculated the coherent wave mixing including the second control pump pulse. In Fig. 10, we show the time-resolved results for the signal at $\theta=0^{\circ}$. With just the pump pulse (dashed), the transmitted probe pulse without pump (dotted) is amplified by a factor of 10 and slightly time-delayed due to the developing stimulation. When the second control pulse is also included

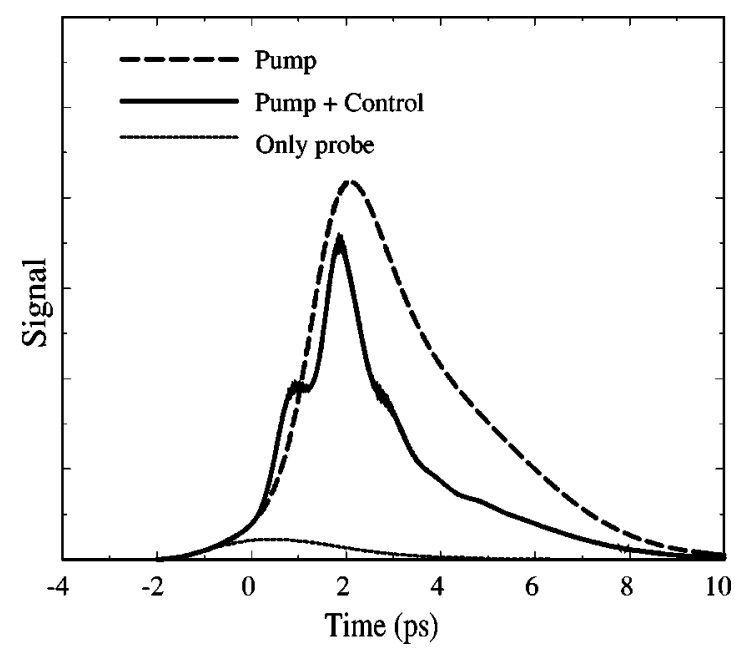

FIG. 10. Coherent control: calculated signal transmission as a function of time for the same parameters as in Fig. 4 for no pump pulses (dotted curve), main pump pulse (dashed curve), and both pump and control pulses (solid curve). The control pump pulse at $1462 \mathrm{meV}$ decreases the gain even though it is nonresonant. The power ratio between pump and control pulses is $\mathrm{I}_{\text {pump }} / \mathrm{I}_{\text {control }}=2$.

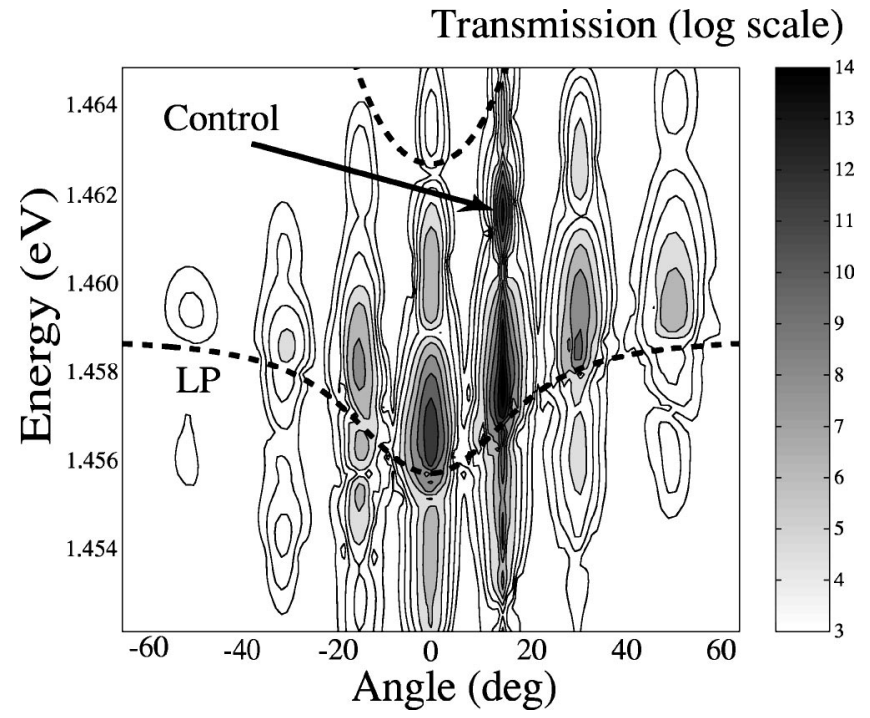

FIG. 11. Computed transmission (log scale) as a function of angle and energy for the same parameters as in Fig. 4, with the addition of the control pulse.

in the calculation, the signal gain decreases by a factor of $\sim 2$. This effect is optimized for simultaneous control and pump pulses as found experimentally. The origin of the gain quenching can be understood by inspecting Fig. 11, which shows the computed angle-resolved transmission spectra. We point out that Fig. 11 is calculated with the same parameters as Fig. 4, where the second control pulse is not included. Remarkably, Fig. 11 shows that the virtual population created by the second pump, produces additional wave-mixing processes, which compete with the normal parametric fission. In fact, we see the appearance of a whole hierarchy of additional spots with respect to Fig. 4. However, unlike the experiments, the predicted idler suppression is comparable to that of the signal. Work is in progress to identify if this is due to higher-order multiple scattering, or coupling to the upper polariton branch, or higher- $k$ excitons. However both the experimental and theoretical results confirm that extreme care has to be taken when exciting these microcavities, since absorption through multiple scattering arises at energies off the main polariton branches.

\section{CONCLUSIONS}

In conclusion, we have presented experimental and theoretical results describing the angle-resolved emission from semiconductor microcavities in the regime of polariton parametric amplification. Above the stimulation threshold, signal and idler modes have a macroscopic and coherent population. Multiple scattering, involving the signal or idler modes, gives rise to additional strong emission beams at wave vectors $-\mathbf{k}_{\mathbf{p}}$ (pump back scattering) and $\mathbf{3} \mathbf{k}_{\mathbf{p}}$ with new spectral peaks. Such emission exhibits a hierarchy of new pairpolariton branches, with their anomalous dispersions lying off branch with respect to the unperturbed lower polariton dispersion. The observed features are well reproduced by equations describing the coherent multiple scattering of the polariton matter modes. Such equations represent a generalization to all $\mathbf{k}$ states of the three mode equations for the 
polariton parametric amplifier. ${ }^{6}$ The nonlinear resonances are also observed in the incoherent luminescence, which allows us to monitor their peculiar angular dispersion, including negative mass pair polaritons. The behavior of the emission branches can be qualitatively described by adopting the Bogulobov scheme to the polariton system in the presence of "condensates" at the pump, signal, and idler wave vectors. The present results give insight into both the nonlinear optics of interacting polariton matter, and the nature of antiHermitian coupling. The development of strong pair scattering in the polariton system, produces these new off-branch dispersions, which will be a general feature of all such strongly excited systems.

\section{ACKNOWLEDGMENTS}

We acknowledge the support of the University of Southampton, Coherent UK Ltd, and Hitachi Europe Ltd. This work was partly supported by HEFCE JR98SOBA, EC CLERMONT HPRN-CT-1999-00132, and EPSRC GR/ N18598. C.C. wishes to thank A. Quattropani, P. Schwendimann, and B. Deveaud for many interesting discussions and their kind support.
*Email address: j.j.baumberg@soton.ac.uk

${ }^{1}$ C. Weisbuch, M. Nishioka, A. Ishikawa, and Y. Arakawa, Phys. Rev. Lett. 69, 3314 (1992).

${ }^{2}$ Le Si Dang, D. Heger, R. Andri, F. Boeuf, and R. Romestain, Phys. Rev. Lett. 81, 3920 (1998).

${ }^{3}$ P. Senellart and J. Bloch, Phys. Rev. Lett. 82, 1233 (1999).

${ }^{4}$ P.G. Savvidis, J.J. Baumberg, R.M. Stevenson, M.S. Skolnick, D.M. Whittaker, and J.S. Roberts, Phys. Rev. Lett. 84, 1547 (2000).

${ }^{5}$ R. Huang, F. Tassone, and Y. Yamamoto, Phys. Rev. B 61, R7854 (2000).

${ }^{6}$ C. Ciuti, P. Schwendimann, B. Deveaud, and A. Quattropani, Phys. Rev. B 62, R4825 (2000).

${ }^{7}$ R. Houdré, C. Weisbuch, R.P. Stanley, U. Oesterle, and M. Ilegems, Phys. Rev. Lett. 85, 2793 (2000).

${ }^{8}$ R.M. Stevenson, V.N. Astratov, M.S. Skolnick, D.M. Whittaker, M. Emam-Ismail, A.I. Tartakovskii, P.G. Savvidis, J.J. Baumberg, and J.S. Roberts, Phys. Rev. Lett. 85, 3680 (2000).

${ }^{9}$ G. Dasbach, T. Baars, M. Bayer, A. Larionov, and A. Forchel,
Phys. Rev. B 62, 13076 (2000).

${ }^{10}$ P.G. Savvidis, J.J. Baumberg, R.M. Stevenson, M.S. Skolnick, D.M. Whittaker, and J.S. Roberts, Phys. Rev. B 62, R13278 (2000).

${ }^{11}$ A.I. Tartakovskii, D.N. Krizhanovskii, and V.D. Kulakovskii, Phys. Rev. B 62, R13298 (2000).

${ }^{12}$ J.J. Baumberg, P.G. Savvidis, R.M. Stevenson, A.I. Tartakovskii, M.S. Skolnick, D.M. Whittaker, and J.S. Roberts, Phys. Rev. B 62, R16247 (2000).

${ }^{13}$ P. Senellart and J. Bloch, Phys. Rev. B 62, R16263 (2000).

${ }^{14}$ C. Ciuti, P. Schwendimann, and A. Quattropani, Phys. Rev. B 63, 041303 (2001).

${ }^{15}$ N.N. Bogolubov, Lectures on Quantum Statistics (Gordon and Breach, New York, 1967).

${ }^{16}$ W.H. Louisell, Coupled Mode and Parametric Electronics (Wiley, New York, 1960).

${ }^{17}$ D.M. Whittaker, Phys. Rev. B 63, 193305 (2001).

${ }^{18}$ A. Kavokin and P. Senellart, private communication.

${ }^{19}$ C. Ell et al., Phys. Rev. Lett. 85, 5392 (2000). 